\section{Dr. Thompson and Dr. Graydon reply}

\section{To the Editor:}

We thank Dr. Bernatsky and colleagues for sharing their work and insightful comments. As discussed in their letter, one of the commonly emerging themes from focus group discussions regarding timely rheumatologic referral is the value of communication between patient, referring physician, and rheumatologist. We believe our study highlights the fact that accurate communication is missing between primary care physicians and rheumatologists. How to best improve communication between these parties is debated.

As clinical educators, we believe that local continuing medical education (CME) is always a rewarding opportunity to deliver a message and create links with the participants. However, global improvement in access to rheumatologic care through CME at the primary care level would likely be inefficient. Effective CME utilizing nondidactic techniques in multiple sequenced sessions is very costly in terms of time and resources.

We are currently pilot-testing a simple one-page rheumatologic referral tool with the goal of improving communication between primary care physicians and rheumatologists. We have sent this tool to 800 local family doctors and have collected 1000 referrals in our database. We are in the preliminary phases of analyzing our data; a few preliminary themes have emerged: (1) Motivating primary care physicians to consistently use the referral tool has been challenging. This strategy was launched with multiple single CME sessions on the use of the referral tool. (2) Despite clearly asking for information, communication remains a problem. Examination of 900 referrals revealed that fewer than $50 \%$ of referring physicians included a simple complete blood count with the referral (when it was clearly asked for).

In what direction should we move? It is clear that rheumatology needs a national strategy to improve the referral process. All rheumatologists mandating referring physicians to use a validated general rheumatology referral form would be a significant step forward in improving the referral process. This strategy would require referring physicians to change behavior, while providing education through repetitive use of the form supported by Web-based and local CME.

ANDY E. THOMPSON, BSc, MD, FRCPC, Assistant Professor of Medicine; SARA L. GRAYDON, MD, Resident in Internal Medicine, University of Western Ontario, London, Ontario, Canada. Address reprint requests to Dr. A.E. Thompson, Rheumatology Centre, St. Joseph's Health Care, 268 Grosvenor Street, PO Box 5777, London, Ontario N6A 4V2.

E-mail: andy.thompson@rogers.com

J Rheumatol 2009;36:4; doi:10.3899/jrheum.081215 